\title{
Angiotensin II Stimulates the NLRP3 Inflammasome to Induce Podocyte Injury and Mitochondrial Dysfunction
}

\author{
Min Zhao ${ }^{a, b}$ Mi Bai ${ }^{a-c}$ Guixia Ding ${ }^{a, b}$ Yue Zhanga, ${ }^{a, b}$ Songming Huang ${ }^{a, b}$ \\ Zhanjun Jia ${ }^{a-c}$ Aihua Zhang ${ }^{a-c}$ \\ ${ }^{a}$ Department of Nephrology, State Key Laboratory of Reproductive Medicine, Children's Hospital of Nanjing Medical \\ University, Nanjing, China; ${ }^{b}$ Jiangsu Key Laboratory of Pediatrics, Nanjing Medical University, Nanjing, China; \\ 'Nanjing Key Laboratory of Pediatrics, Nanjing, China
}

\section{Keywords}

Angiotensin II - Mitochondrial dysfunction · NLRP3

inflammasome Podocyte

\begin{abstract}
Background: We previously reported that the NLRP3 inflammasome played an important role in mediating the podocyte injury induced by aldosterone. However, more studies on the role of the NLRP3 inflammasome in the pathogenesis of podocytopathy are still required. The present study was undertaken to study the role of the NLRP3 inflammasome in angiotensin II (Ang II)-induced podocyte injury, as well as the potential mechanisms. Methods: In this study, we used an Ang II infusion model in $\mathrm{NLRP3}^{-/-}$mice. In cultured podocytes, we used siRNA to silence NLRP3; then we treated the podocytes with Ang II. Results: Following Ang II treatment, we found that the NLRP3 inflammasome was significantly activated in line with mitochondrial dysfunction in a doseand time-dependent manner. Silencing NLRP3 by siRNA transfection ameliorated podocyte apoptosis, attenuated the loss of the podocyte proteins nephrin and podocin, and protected mitochondrial function. Ang II infusion activated the NLRP3 inflammasome, caused albuminuria, and induced
\end{abstract}

(c) 2018 S. Karger AG, Basel

E-Mail karger@karger.com www.karger.com/kdd podocyte damage, which was all blocked in the $\mathrm{NLRP}^{-/-}$ mice. At the same time, NLRP3 deletion also ameliorated the mitochondrial dysfunction induced by Ang II infusion. However, the deletion of NLRP3 did not affect the Ang II hypertension. Conclusion: Taken together, these results demonstrate an important role of the NLRP3 inflammasome in mediating Ang II-induced podocyte injury and mitochondrial dysfunction, suggesting that the NLRP3 inflammasome might be an effective therapeutic target against podocytopathy.

(c) 2018 S. Karger AG, Basel

\section{Introduction}

Angiotensin II (Ang II), a major bioactive product of the renin-angiotensin system, plays a critical role in maintaining blood pressure and fluid homeostasis $[1,2]$. However, Ang II also contributes to the pathogenesis of various diseases, mainly by inducing oxidative stress, inflammation, cholesterol accumulation, and fibrosis [3-5]. Moreover, accumulating evidence shows that inflammation plays an important role in the development and pro-

Min Zhao and Mi Bai contributed equally to this work. 
gression of renal diseases [6-8]. For example, a previous study reported that Ang II could induce inflammation in renal tubular cells [9].

The NLRP3 (NOD-like receptor 3) inflammasome is well characterized among the members of the NLR family. A functional inflammasome complex is formed through CARD-CARD interaction of ASC (apoptosis-associated speck-like protein containing carboxy-terminal CARD) with procaspase-1. Activation of the NLRP3 inflammasome finally triggers the maturation of IL- $1 \beta$ and IL-18 via activated caspase- $1[10,11]$. Studies from our and other groups suggest that activation of the NLRP3 inflammasome is involved in renal tubular and podocyte injury induced by albuminuria or aldosterone [12-15]. However, the role of the NLRP3 inflammasome in Ang II-induced podocyte injury has not been defined.

Mitochondria are complex intracellular organelles that are responsible for various metabolic activities including energy production via oxidative phosphorylation. Mitochondrial dysfunction is characterized by increased reactive oxygen species production, the accumulation of impaired mtDNA, and progressive respiratory chain dysfunction. Growing evidence indicates that mitochondrial dysfunction is involved in podocyte injury [16]. In agreement with these results, we found in our previous study [17] that mitochondrial dysfunction is an early event in podocyte injury. Recently, a study reported Ang II could induce mitochondrial dysfunction in skeletal muscle [18]. However, the role of mitochondrial dysfunction in Ang II-induced podocyte injury remains elusive.

In the present study, using a siRNA approach and $\mathrm{NLRP3}^{-/-}$mice, we show that NLRP3 inflammasome activation contributes to Ang II-induced podocyte injury and mitochondrial dysfunction. These findings not only reveal a novel mechanism mediating Ang II-induced podocyte injury but also offer a new target for the treatment of podocytopathy.

\section{Methods}

\section{Reagents and Antibodies}

Newborn bovine serum was purchased from Gibco (Gibco, USA). Ang II and murine interferon- $\gamma$ were obtained from SigmaAldrich (St. Louis, MO, USA). Antibodies against NLRP3, podocin, and nephrin were from Abcam (Cambridge, MA, USA). Anticaspase- 1 and anti- $\beta$-actin antibodies were from Santa Cruz Biotechnology (Santa Cruz, CA, USA).

\section{Podocyte Culture}

Conditionally immortalized murine MPC5 podocyte clonal cells (kindly provided by Peter Mundel at the Icahn School of Med-
Table 1. Primer sequences for quantitative real-time PCR

\begin{tabular}{ll}
\hline Gene symbol & Primer sequence 5' $^{\prime}{ }^{\prime}$ \\
\hline NLRP3 & $\begin{array}{l}\text { GTGGTGACCCTCTGTGAGGT } \\
\text { TCTTCCTGGAGCGCTTCTAA }\end{array}$ \\
\hline Caspase-1 & ACAAGGCACGGGACCTATG \\
& TCCCAGTCAGTCCTGGAAATG \\
\hline Nephrin & ATGGGAGCTAAGGAAGCCACA \\
& CCACACCACAGCTTAACTGTC \\
\hline Podocin & TGCTACTACCGCATGGAAAATG \\
\hline mtDNA & CTGCATCTAAGGCAACCTTTACA \\
\hline ATS rRNA & ACCGGTAGGAATTGCGATAA \\
\hline GAPDH & TTCGGAACTGAGGCCATGATT \\
& TTTCGCTCTGGTCCGTCTTG \\
\hline
\end{tabular}

icine at Mount Sinai through Dr. Jie Ding at Peking University) were cultured and induced to differentiate as described previously [19]. The cells were maintained in RPMI 1640 medium containing $10 \%$ heat-inactivated fetal calf serum, $100 \mathrm{U} / \mathrm{mL}$ penicillin $\mathrm{G}, 10$ $\mathrm{U} / \mathrm{mL}$ recombinant murine interferon- $\gamma$, and $100 \mathrm{mg} / \mathrm{mL}$ streptomycin in a $5 \% \mathrm{CO}_{2}$ atmosphere at $33^{\circ} \mathrm{C}$. Podocytes were maintained without interferon- $\gamma$ at $37^{\circ} \mathrm{C}$ for $10-14$ days to induce differentiation before the experiments.

\section{siNLRP3 Transfection}

NLRP3 siRNA and vehicle siRNA were from GenePharma (China). Podocytes were cultivated to $40-60 \%$ confluence in culture medium containing no penicillin or streptomycin. The cells were transfected with 50 nM NLRP3 siRNA or control siRNA $24 \mathrm{~h}$ before Ang II treatment using siRNA-Mate (GenePharma) according to the manufacturer's instructions. The siRNA sequences were as follows: siNLRP3, $5^{\prime}$-CGGCCUUACUUCAAUCUGUTT- $3^{\prime}$ and $5^{\prime}$-ACAGAUUGAAGUAAGGCCGTT- $3^{\prime}$.

Animals

The study protocols were reviewed and approved by the Institutional Animal Care and Use Committee at Nanjing Medical University, China. Nlrp3 $3^{-/-}$mice on a C57BL/6J background were purchased from The Jackson Laboratory (Sacramento, CA, USA). In brief, heterozygous littermates were bred to generate homozygous KO mice and wild-type (WT) littermate controls; 10- to 12-weekold male mice $(22-26 \mathrm{~g})$ were used in our study. All mice were maintained on a 12-h light-dark cycle in a temperature-controlled $\left(19-21^{\circ} \mathrm{C}\right)$ room and were fed standard rodent chow.

\section{Animal Model of Ang II Infusion}

Male NLRP3 ${ }^{-/-}(\mathrm{KO})$ and WT mice aged 10-12 weeks and weighing 22-26 g were treated with Ang II using subcutaneously implanted osmotic minipumps (ALZET; DURECT, Cupertino, 


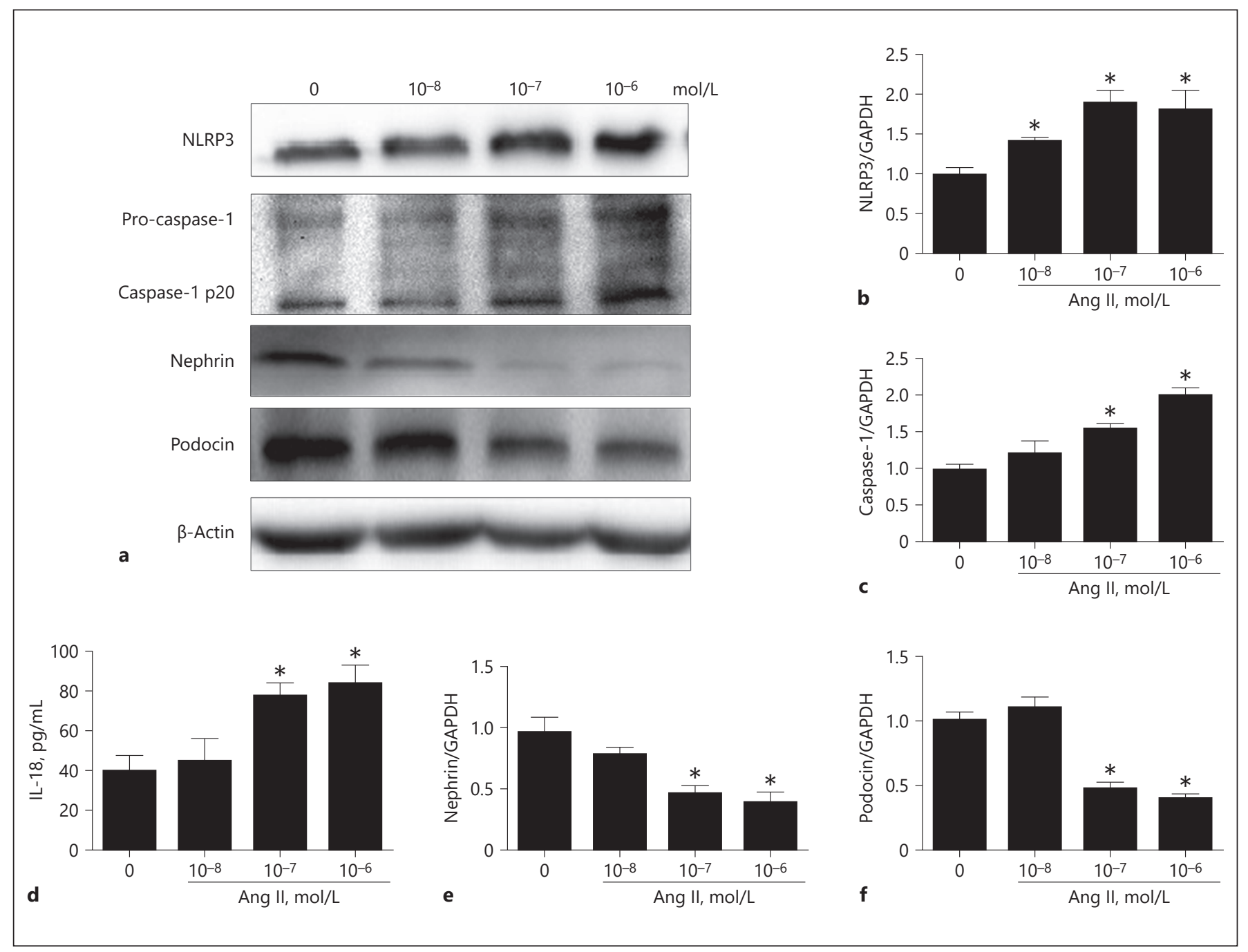

Fig. 1. Angiotensin II (Ang II) activated the NLRP3 inflammasome and induced podocyte injury. Cells were grown on 6-well plates until $80 \%$ confluence, then treated with different doses of Ang II $\left(10^{-8}, 10^{-7}\right.$, and $\left.10^{-6} \mathrm{~mol} / \mathrm{L}\right)$ for $24 \mathrm{~h}$. a Western blotting analyses of NLRP3, caspase-1, nephrin, and podocin expression. b, c qRT-
PCR analysis of NLRP3 and caspase- 1 expression. d ELISA analysis of IL-18 levels in the supernatant of cultured cells. e, f qRT-PCR analyses of nephrin and podocin expression. Data are presented as means $\pm \operatorname{SE}(n=6) .{ }^{*} p<0.05$ vs. control $(0 \mathrm{~mol} / \mathrm{L})$.
CA, USA). The pumps were placed by making an incision in the right flank region under light anesthesia with 3\% isoflurane; they delivered a continuous infusion of Ang II (300 ng/ $\mathrm{kg} / \mathrm{min})$ for 28 days. All mice were maintained on a 12 -h light-dark cycle in a temperature-controlled $\left(19-21^{\circ} \mathrm{C}\right)$ room. They were fed standard rodent chow and had free access to drinking water.

\section{Apoptosis Analysis}

Hoechst 33258 Staining. Podocytes were grown on glass coverslips for measurement of apoptosis. After treatment, the podocytes were stained with Hoechst 33258 and viewed by fluorescence microscopy.
Annexin V-Fluorescein Isothiocyanate Conjugated with Propidium Iodide. After treatment, the podocytes were seeded into 6-well plates, and apoptosis was quantified by flow cytometry using annexin $\mathrm{V}$-fluorescein isothiocyanate and propidium iodide double staining (annexin V-fluorescein isothiocyanate apoptosis Q18 detection kit; BD Biosciences), according to the manufacturer's instructions.

TUNEL Assay in Cultured Podocytes. Apoptotic cell death was determined using TUNEL staining with an In Situ Cell Death Detection Kit (Roche Molecular Biochemicals, Mannheim, Germany), following the manufacturer's protocol. Morphological changes in podocytes undergoing apoptosis were then detected by counterstaining them with Hoechst 33258. The slides were examined by confocal microscopy. 


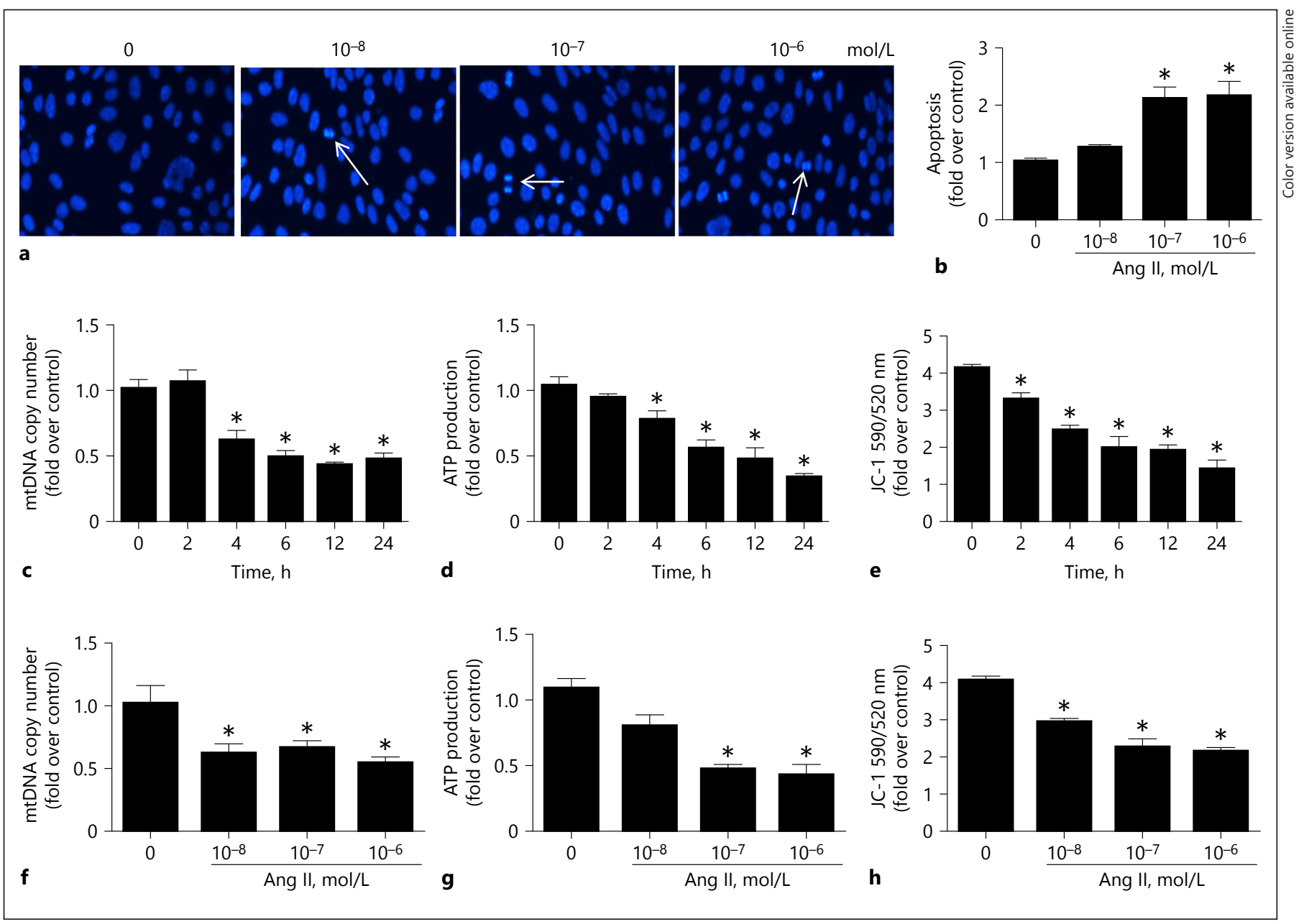

Fig. 2. Angiotensin II (Ang II) induced podocyte apoptosis and mitochondrial dysfunction. Cells were grown on 6-well plates until $80 \%$ confluence, then treated with Ang II $\left(10^{-6} \mathrm{~mol} / \mathrm{L}\right)$ for different periods of time $(0,2,4,6,12$, and $24 \mathrm{~h})$ or at different doses of Ang II. a Hoechst 33258 staining. The arrows indicate chroma- tin condensation and fragmentation. b Cell apoptosis after Ang II treatment detected by flow cytometry. c, $\mathbf{f}$ qRT-PCR analysis of mtDNA copy number. $\mathbf{d}, \mathbf{g}$ ATP production. e, $\mathbf{h}$ Quantitation of JC-1 fluorescence by flow cytometry. Data are presented as means \pm SE $(n=6) .{ }^{*} p<0.05$ vs. control $(0 \mathrm{~mol} / \mathrm{L})$.

\section{Western Blotting}

Podocytes or renal tissue were lysed in protein lysis buffer (50 $\mathrm{mmol} / \mathrm{L}$ Tris, $150 \mathrm{mmol} / \mathrm{L} \mathrm{NaCl}, 10 \mathrm{mmol} / \mathrm{L}$ EDTA, 1\% Triton $\mathrm{X}-100,200 \mathrm{mmol} / \mathrm{L}$ sodium fluoride, and $4 \mathrm{mmol} / \mathrm{L}$ sodium orthovanadate as protease inhibitors; $\mathrm{pH}$ 7.5) for $15 \mathrm{~min}$ on ice. Protein was extracted. Immunoblotting was performed with primary antibodies against NLRP3 (1:500), nephrin $(1: 1,000)$, podocin (1: $1,000)$, caspase- $1(1: 250)$, or $\beta$-actin $(1: 1,000)$, followed by the addition of horseradish peroxidase-labelled secondary antibodies. The blots were visualized with Amersham ECL Detection Systems (Amersham, UK). Densitometric analysis was performed using Quantity One Software (Bio-Rad).
Quantitative Real-Time PCR and Reverse Transcription PCR

Total RNA was isolated from MPC cells and the renal cortex using a TRIzol Total RNA Isolation Kit (Invitrogen) according to the manufacturer's protocol. The RNA was eluted with RNase-free water. Reverse transcription was performed using the Superscript III RT Kit (Invitrogen) according to the manufacturer's protocols. Briefly, the reactions were incubated at $65^{\circ} \mathrm{C}$ for 5 min and then at $50^{\circ} \mathrm{C}$ for $60 \mathrm{~min}$. Total DNA from cultured podocytes and renal tissue were extracted with a DNeasy Tissue Kit (QIAGEN Science). Oligonucleotides were designed with Primer3 software (available at http://frodo.wi.mit.edu/cgi-bin/primer3/primer3_www.cgi) and synthesized at Invitrogen (the primer sequences are shown in Table 1). Real-time PCR amplification was performed using the SYBR Green Master Mix (Roche) and the Prism 7500 Real-Time PCR Detection System (Applied Biosystems). The cycling condi- 


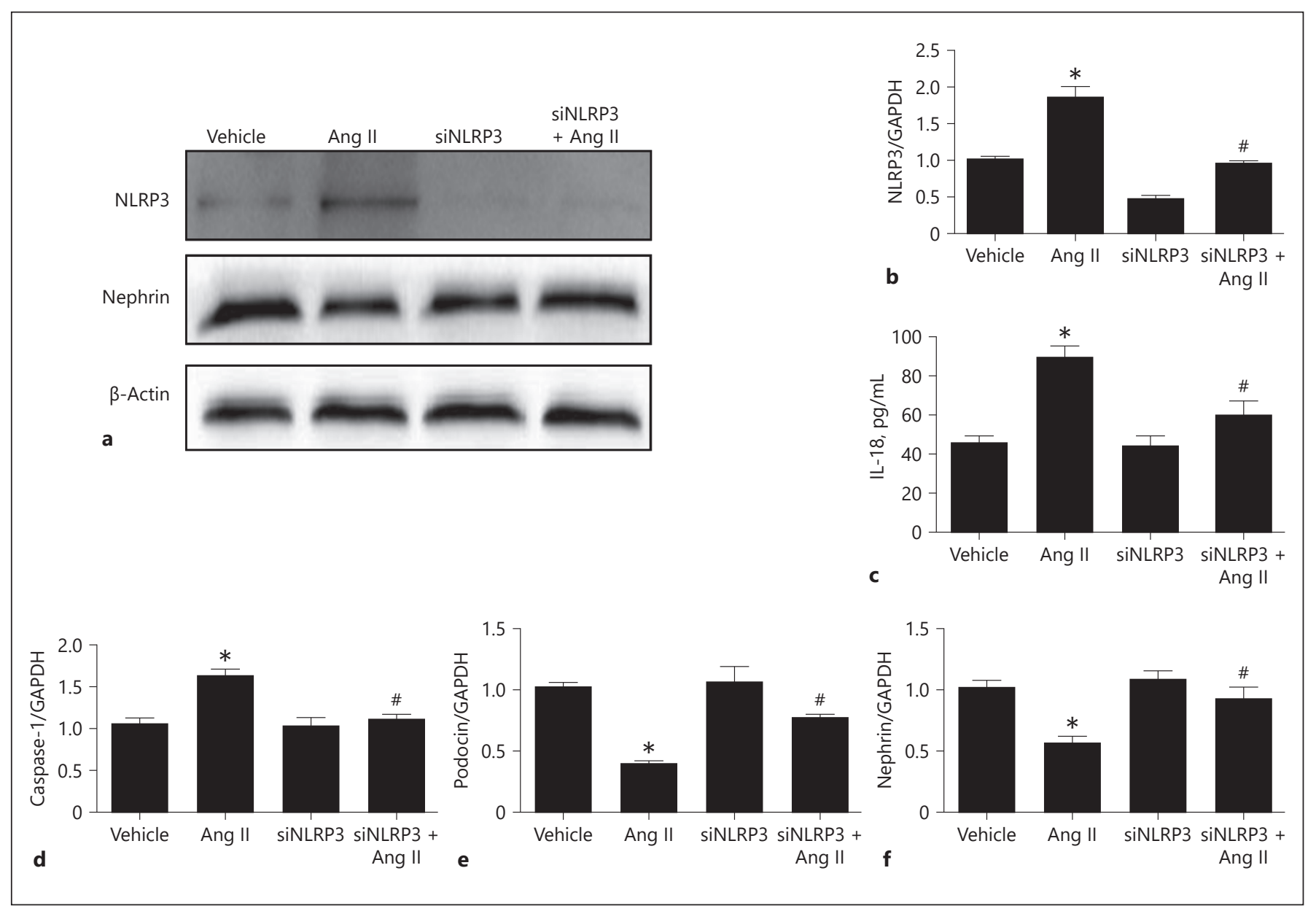

Fig. 3. Silencing NLRP3 protected angiotensin II (Ang II)-induced podocyte injury. Cells were grown on 6-well plates until 30\% confluence and transfected with siNLRP3 for $24 \mathrm{~h}$, then treated with Ang II $\left(10^{-6} \mathrm{~mol} / \mathrm{L}\right)$ for another $24 \mathrm{~h}$. a Western blotting analysis of NLRP3 and nephrin. $\mathbf{b}$ qRT-PCR analysis of NLRP3. $\mathbf{c}$ ELISA analysis of the IL-18 concentration in the medium of cultured cells.

tions were $95^{\circ} \mathrm{C}$ for $10 \mathrm{~min}$ followed by 40 repeats of $95^{\circ} \mathrm{C}$ for 15 $\mathrm{s}$ and $60^{\circ} \mathrm{C}$ for $1 \mathrm{~min}$. Relative amounts of mtDNA copy numbers were normalized to $18 \mathrm{~S}$ ribosomal RNA levels encoded by the nuclear DNA, and mRNA was normalized to GAPDH and calculated using the delta-delta method from threshold cycle numbers.

\section{ATP Content Measurement}

ATP levels in the podocytes were determined with a luciferasebased bioluminescence assay kit (Sigma-Aldrich) in a FLUOstar Optima reader according to the manufacturer's instructions.

\section{Mitochondrial Membrane Potential}

The mitochondrial membrane potential (MMP) of podocytes was monitored using JC-1, a MMP-sensitive fluorescent dye, as described previously [20]. Briefly, podocytes were washed twice with PBS and incubated in the dark with $\mathrm{JC}-1$ probe $(7.5 \mathrm{mmol} / \mathrm{L}$; $30 \mathrm{~min}$ at $37^{\circ} \mathrm{C}$ ). The cells were washed with JC-1 washing buffer,
Podocytes were incubated with Ang II for $24 \mathrm{~h}$, and the medium was collected to detect IL- 18 by ELISA. $\mathbf{d}$ qRT-PCR analysis of caspase-1. e qRT-PCR analysis of podocin. $\mathbf{f}$ qRT-PCR analysis of nephrin. Data are presented as means \pm SE $(n=6) .{ }^{*} p<0.05$ vs. vehicle group; ${ }^{\#} p<0.05$ vs. Ang II-treated group.

and fluorescence was detected by flow cytometry. The relative MMP was calculated using the ratio of J-aggregate/monomer $(590 / 520 \mathrm{~nm})$. Values are expressed as fold change in J-aggregate/ monomer fluorescence over control cells.

\section{Blood Pressure Measurement}

Systolic blood pressure of the mice was measured by the tailcuff method, using a Visitech BP-2000 Blood Pressure Analysis System (Visitech Systems, Apex, NC, USA).

\section{Transmission Electron Microscopy}

Fresh kidney tissues were fixed in $1.25 \%$ glutaraldehyde/0.1 $\mathrm{mol} / \mathrm{L}$ phosphate buffer and postfixed in $1 \% \mathrm{OsO}_{4} / 0.1 \mathrm{~mol} / \mathrm{L}$ phosphate buffer. Ultrathin sections $(60 \mathrm{~nm})$ were cut on a microtome, placed on copper grids, stained with uranyl acetate and lead citrate, and examined under an electron microscope (JEM-1010; JEOL Ltd., Tokyo, Japan). 


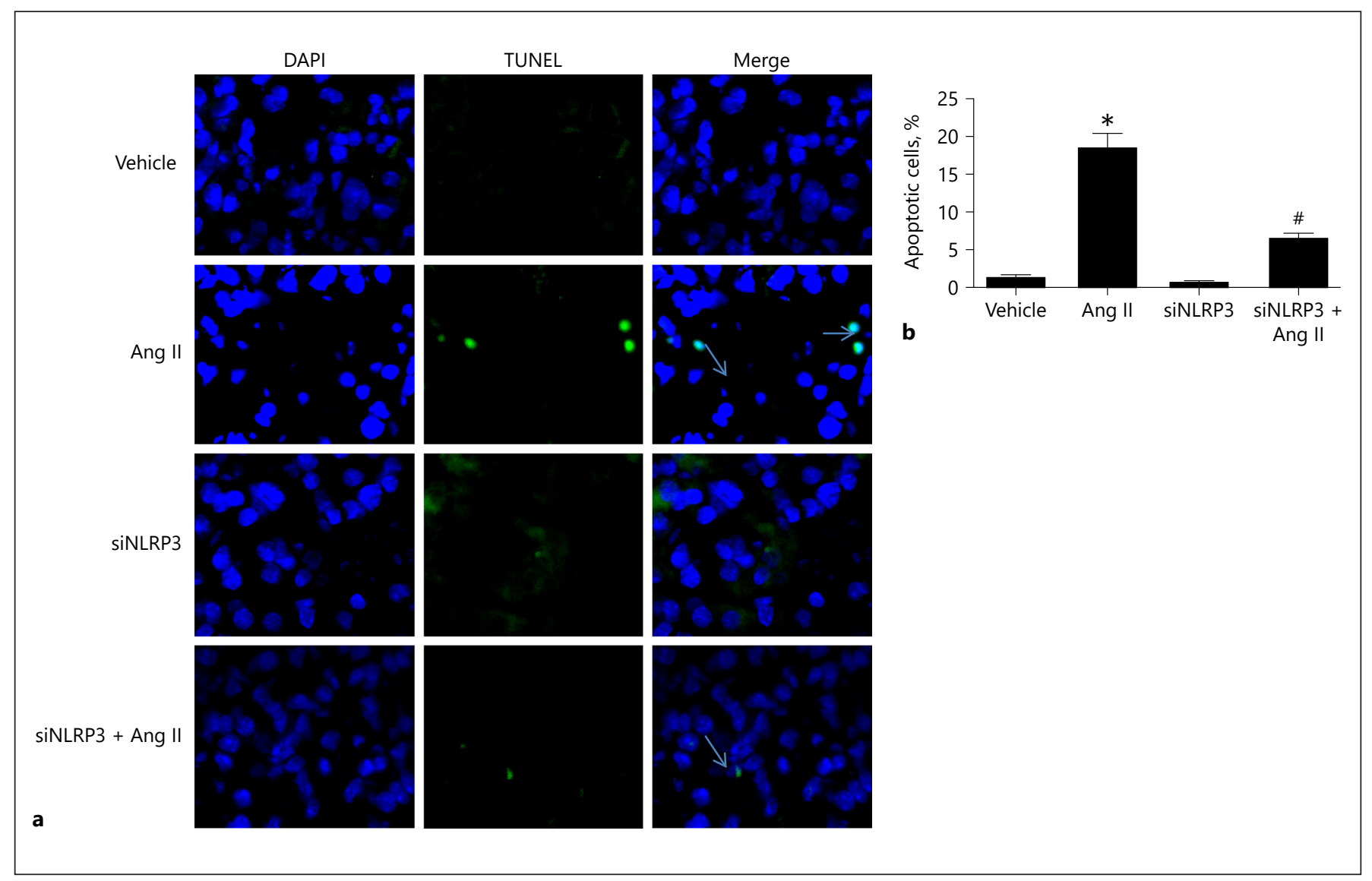

Fig. 4. Silencing of NLRP3 attenuated angiotensin II (Ang II)-induced podocyte apoptosis. Cells were grown on 6-well plates until $30 \%$ confluence and transfected with siNLRP3 for $24 \mathrm{~h}$, then treated with Ang II $\left(10^{-6} \mathrm{~mol} / \mathrm{L}\right)$ for another 24 h. a TUNEL staining.
The arrows indicate TUNEL-positive signals. b Quantitation of podocyte apoptosis, which was determined by flow cytometry. Data are presented as means $\pm \operatorname{SE}(n=6) .{ }^{*} p<0.05$ vs. vehicle group; ${ }^{*} p<0.05$ vs. Ang II-treated group.

\section{Analysis of Urinary Albumin}

The urinary concentration of albumin was determined using enzyme-linked immunosorbent assay kits from Exocell (Philadelphia, PA, USA).

\section{Statistical Analysis}

Data are presented as means \pm SE. Statistical analysis was performed using ANOVA followed by a Bonferroni posttest or unpaired Student $t$ test. $p<0.05$ was considered statistically significant.

\section{Results}

\section{Ang II Activated the NLRP3 Inflammasome and}

Induced Podocyte Injury

We firstly examined the effect of Ang II on activation of the NLRP3 inflammasome. As shown by the data, Ang II induced NLRP3 inflammasome activation as evidenced by the increments in NLRP3, active caspase-1, and IL-18 in a dose-dependent manner (Fig. 1a-d). Meanwhile, Ang II dose-dependently downregulated the expression of the podocyte proteins nephrin and podocin (Fig. 1e, f) accompanied by enhanced podocyte apoptosis (Fig. 2a, b). These results suggest that NLRP3 inflammasome activation might be involved in Ang II-induced podocyte injury.

\section{Ang II Induced Mitochondrial Dysfunction in Podocytes}

To evaluate the effect of the Ang II challenge on mitochondrial function, we assessed the MMP, ATP levels, and mtDNA copy number. As shown in Figure $2 c-h$, Ang II could induce a reduction in mtDNA copy number, ATP content, and MMP in podocytes in a dose- and timedependent manner. These results demonstrate that Ang II induced mitochondrial dysfunction in podocytes. 


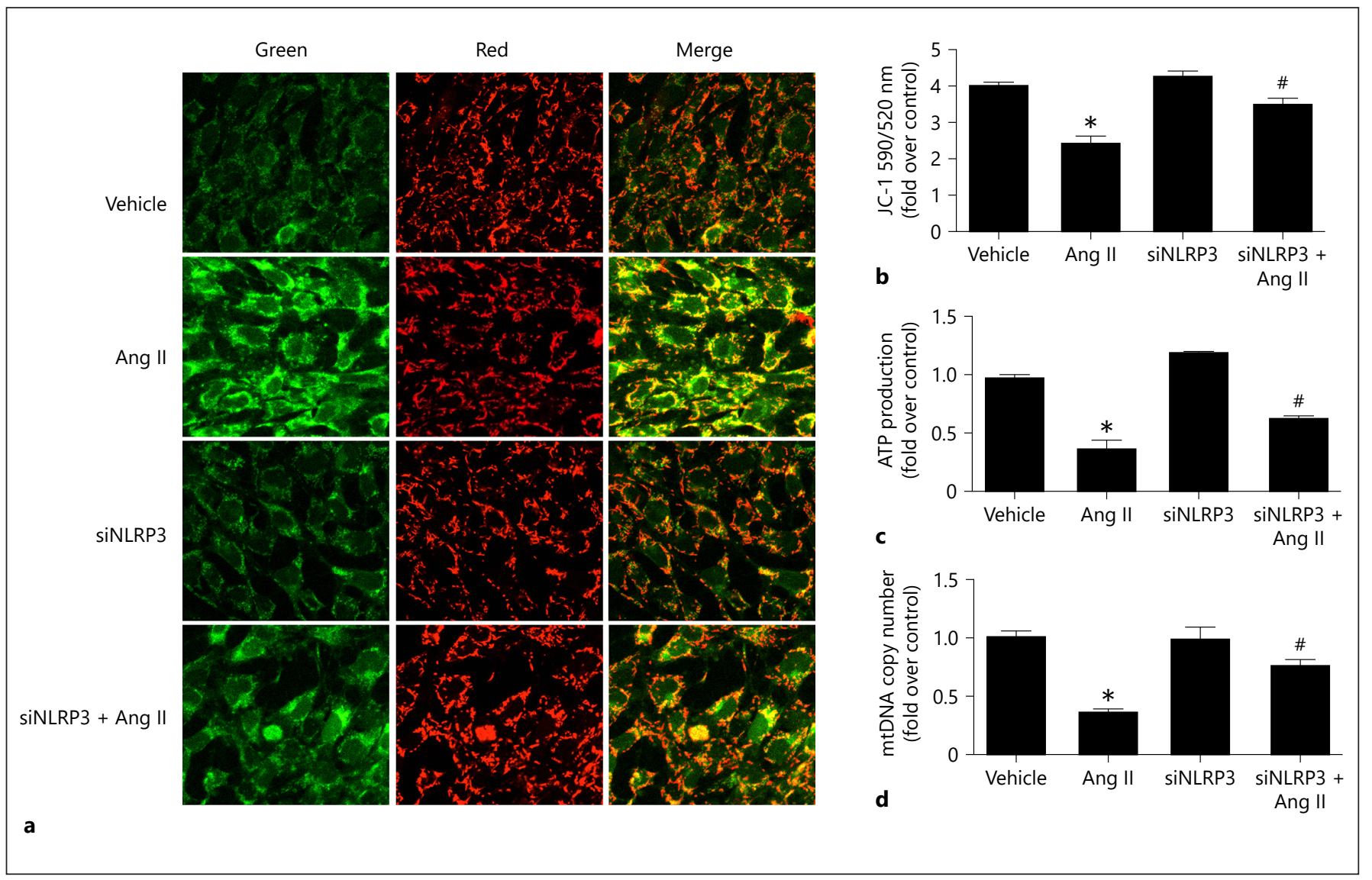

Fig. 5. Knockdown of NLRP3 attenuated angiotensin II (Ang II)induced mitochondrial dysfunction. Cells were grown on 6-well plates until 30\% confluence and transfected with siNLRP3 for 24 $\mathrm{h}$, then treated with Ang II $\left(10^{-6} \mathrm{~mol} / \mathrm{L}\right)$ for another $24 \mathrm{~h}$. a Representative images of JC-1 staining. b Quantitation of JC-1 fluores- cence by flow cytometry. c ATP content was detected as described in the Methods section. $\mathbf{d}$ qRT-PCR analysis of the mtDNA copy number. Data are presented as means \pm SE $(n=6) .{ }^{*} p<0.05$ vs. vehicle group; ${ }^{\#} p<0.05$ vs. Ang II-treated group.

\section{Silencing NLRP3 Attenuated Ang II-Induced}

Mitochondrial Dysfunction in Podocytes

We then explored the effect of NLRP3 on mitochondrial dysfunction in podocytes challenged with Ang II. As shown in Figure 5, Ang II strikingly impaired mitochondrial function, which was partially but significantly ameliorated by siNLRP3 as evidenced by restoration of the MMP, ATP content, and mtDNA copy number, indicating that Ang II-induced mitochondrial dysfunction in podocytes might be mediated by activation of the NLRP3 inflammasome.

Deletion of NLRP3 Improved Ang II-Induced Podocyte Injury and Mitochondrial Dysfunction in Mice

We further investigated whether deletion of NLRP3 could inhibit Ang II-induced podocyte injury and mito- 


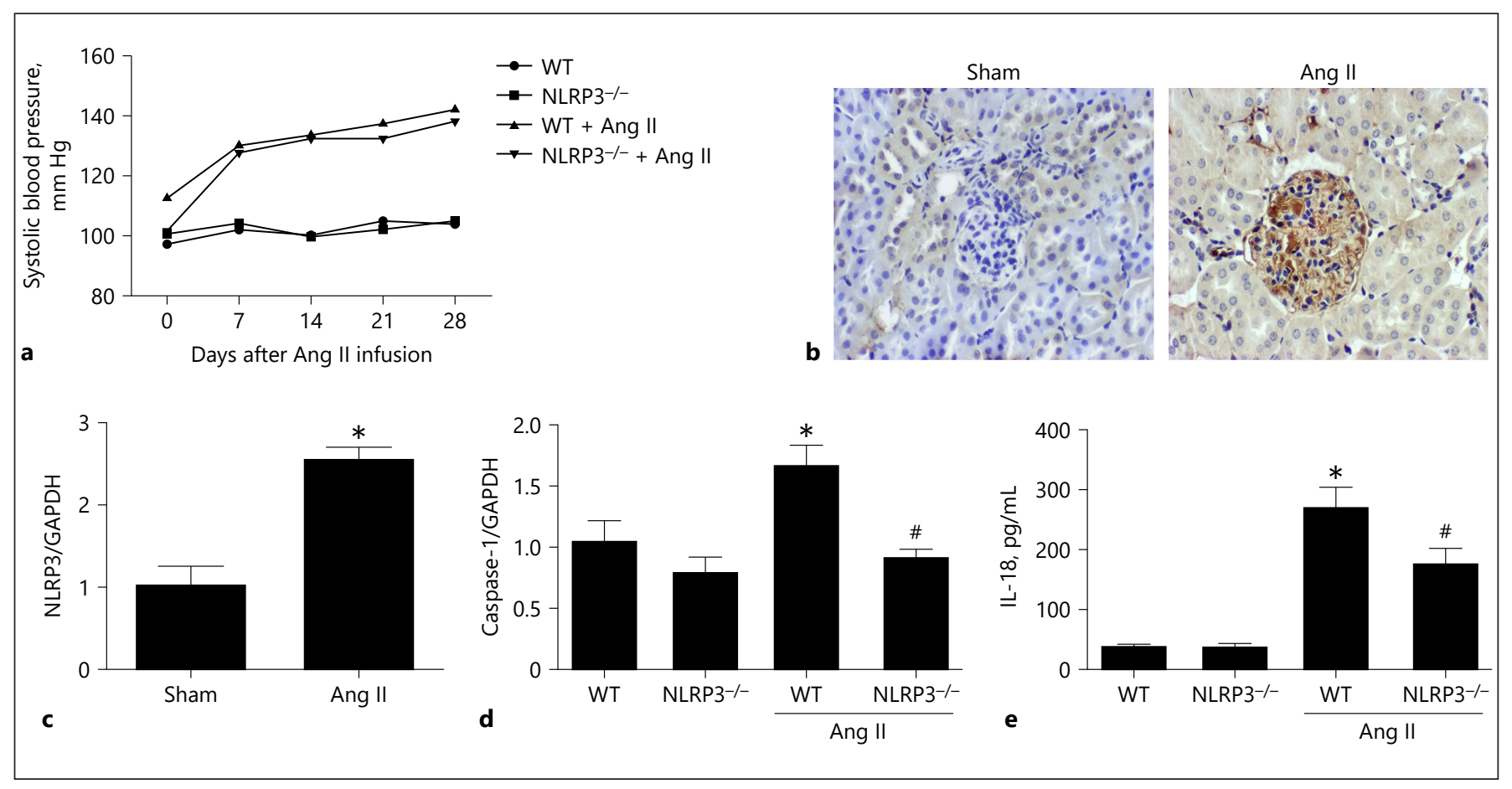

Fig. 6. Deletion of NLRP3 blocked activation of the NLRP3 inflammasome in angiotensin II (Ang II)-treated murine kidneys. a Systolic blood pressure after Ang II infusion in wild-type (WT) and $\mathrm{NLRP}^{-/-}$mice determined by the tail-cuff method. b Immunohistochemistry of NLRP3 in the kidneys. c qRT-PCR analysis of

chondrial dysfunction in mice. First, we measured the blood pressure using the tail-cuff method. As shown in Figure 6a, both genotypes displayed a similar hypertensive response after Ang II infusion. At the same time, Ang II infusion enhanced the expression of NLRP3 in glomeruli (Fig. 6b, c) in line with increments in caspase- 1 in the kidneys (Fig. 6d) and IL-18 in the blood (Fig. 6e). Strikingly, NLRP3 deletion blocked the upregulation of renal caspase- 1 and the increase in blood IL-18 levels (Fig. 6d, e), suggesting a blockade of NLRP3 inflammasome activation.

Next, we found that the output of urinary albumin was significantly blunted in NLRP $3^{-/-}$mice as compared with the WT controls following Ang II infusion for 28 days (Fig. 7a). Consistent with the attenuation of albuminuria, we observed that the reduction of podocin and nephrin was partly reversed in $\mathrm{NLRP}^{-/-}$mice (Fig. $7 \mathrm{c}-\mathrm{e}$ ), and the podocyte foot process fusion determined by electron microscopy was also mitigated in $\mathrm{NLRP}^{-/-}$mice following 4-week Ang II infusion (Fig. 7b).

Finally, we observed the mitochondrial morphology of the podocytes in kidneys of mice after 4-week Ang II in-
NLRP3 in the kidneys. d qRT-PCR analysis of caspase-1 in the kidneys. e IL-18 levels in the blood. Data are presented as means \pm $\mathrm{SE}(n=8) .{ }^{*} p<0.05$ vs. vehicle group; ${ }^{*} p<0.05$ vs. Ang II-treated group.

fusion, as well as the mitochondrial DNA copy number. As shown in Figure 8, $\mathrm{NLRP}^{-/-}$mice exhibited an obvious improvement of the abnormality of their mitochondrial morphology and of the loss in mtDNA copy number. Taken together, these in vivo data demonstrate that deletion of NLRP3 in mice protects the podocytes against Ang II-induced injury, possibly to some extent through a mitochondrial mechanism.

\section{Discussion}

Podocyte injury occurs in all glomerular diseases [21]. In the present study, using cells and animals, we investigated the contribution of the NLRP3 inflammasome in the pathogenesis of podocyte injury and mitochondrial dysfunction resulting from Ang II infusion. The findings indicated that NLRP3 inflammasome activation was attributable to the Ang II-induced podocyte apoptosis, loss of the slit diaphragm proteins nephrin and podocin, and mitochondrial dysfunction, which would lead to proteinuria. 


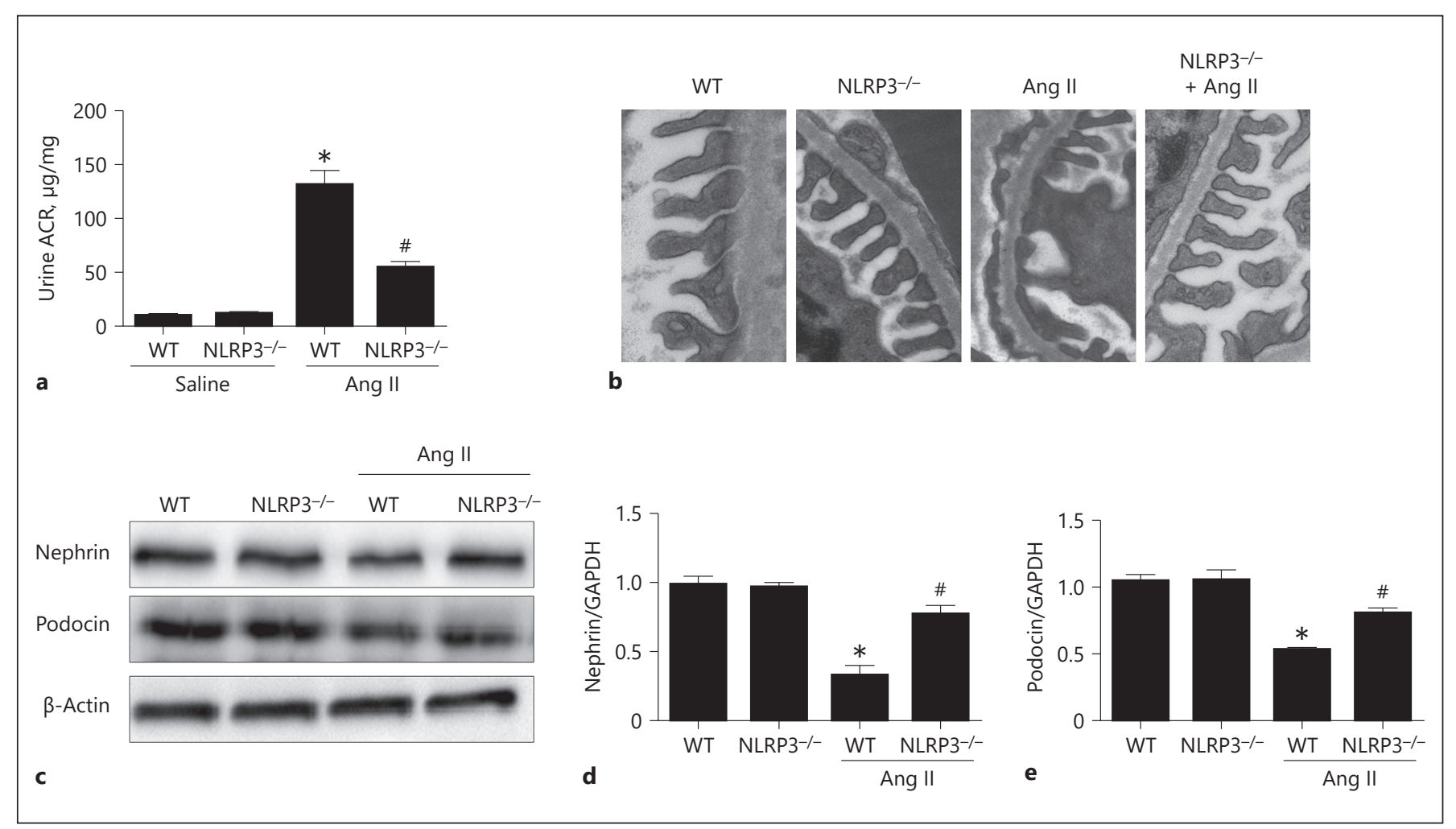

Fig. 7. Deletion of NLRP3 attenuated angiotensin II (Ang II)-induced kidney injury. a Urinary albumin output. b Representative images of podocyte foot process morphology by electron microscopy. c Western blotting analysis of nephrin and podocin. $\mathbf{d}$ qRT-
PCR analysis of nephrin. e qRT-PCR analysis of podocin. Data are presented as means \pm SE $(n=8) .{ }^{*} p<0.05$ vs. vehicle group; ${ }^{\#} p<$ 0.05 vs. Ang II-treated group. ACR, albumin/creatinine ratio; WT, wild type.
Ang II, as the strongest effector molecule in the RAS system, was reported to be involved in the pathogenesis of various kidney diseases through inducing renal inflammation $[22,23]$. Recent evidence demonstrated that the NLRP3 inflammasome played a detrimental role in kidney diseases [24-26]. Our previous study also showed that the NLRP3 inflammasome was involved in aldosterone-induced podocyte injury and 5/6 nephrectomyrelated renal tubular damage $[12,13,27]$. In the present study, we found that Ang II activated the NLRP3 inflammasome in podocytes and that NLRP3 silencing attenuated the Ang II-induced podocyte apoptosis and loss of the podocyte-specific proteins nephrin and podocin. In vivo, albuminuria was partly mitigated in $\mathrm{NLRP}^{-/-}$mice, which is in agreement with the finding by Wen et al. [28]. We further found that the loss of nephrin and podocin and the podocyte foot process fusion were ameliorated in $\mathrm{NLRP}^{-/-}$mice. These data highly suggest a pathogenic role of the NLRP3 inflammasome in Ang II-mediated podocyte injury. However, systemic deletion of NLRP3 could not fully address the contribution of podocyte NLRP3 in mediating the Ang II-induced podocyte injury. In the future, studies in animals with podocyte-specific deletion of NLRP3 are still needed.

Over the past decade, many studies have shown that Ang II can activate NADPH oxidases and increase reactive oxygen species production to further participate in different pathophysiological processes including the promotion of mitochondrial dysfunction [29-31]. However, the exact mechanism is not yet conclusively elucidated. Previous studies from our and other groups demonstrated that albumin-induced mitochondrial dysfunction was mediated by NLRP3 inflammasome activation in tubular epithelial cells $[14,32]$. However, the relationship between mitochondrial dysfunction and activation of the NLRP3 inflammasome in response to an Ang II challenge in podocytes remains unclear. In cells, our data showed that mitochondrial function was significantly impaired in podocytes by Ang II as demonstrated by reduction of the mtDNA copy number, ATP content, and MMP. Knock- 
Fig. 8. Deletion of NLRP3 protected against angiotensin II (Ang II)-induced mitochondrial dysfunction in the kidneys of mice. a Representative images of mitochondrial morphology in podocytes by electron microscopy. $\mathbf{b}$ qRT-PCR of the mtDNA copy number. Data are presented as means \pm SE $(n=8) .{ }^{*} p<0.05$ vs. vehicle group; ${ }^{\#} p<$ 0.05 vs. Ang II-treated group.

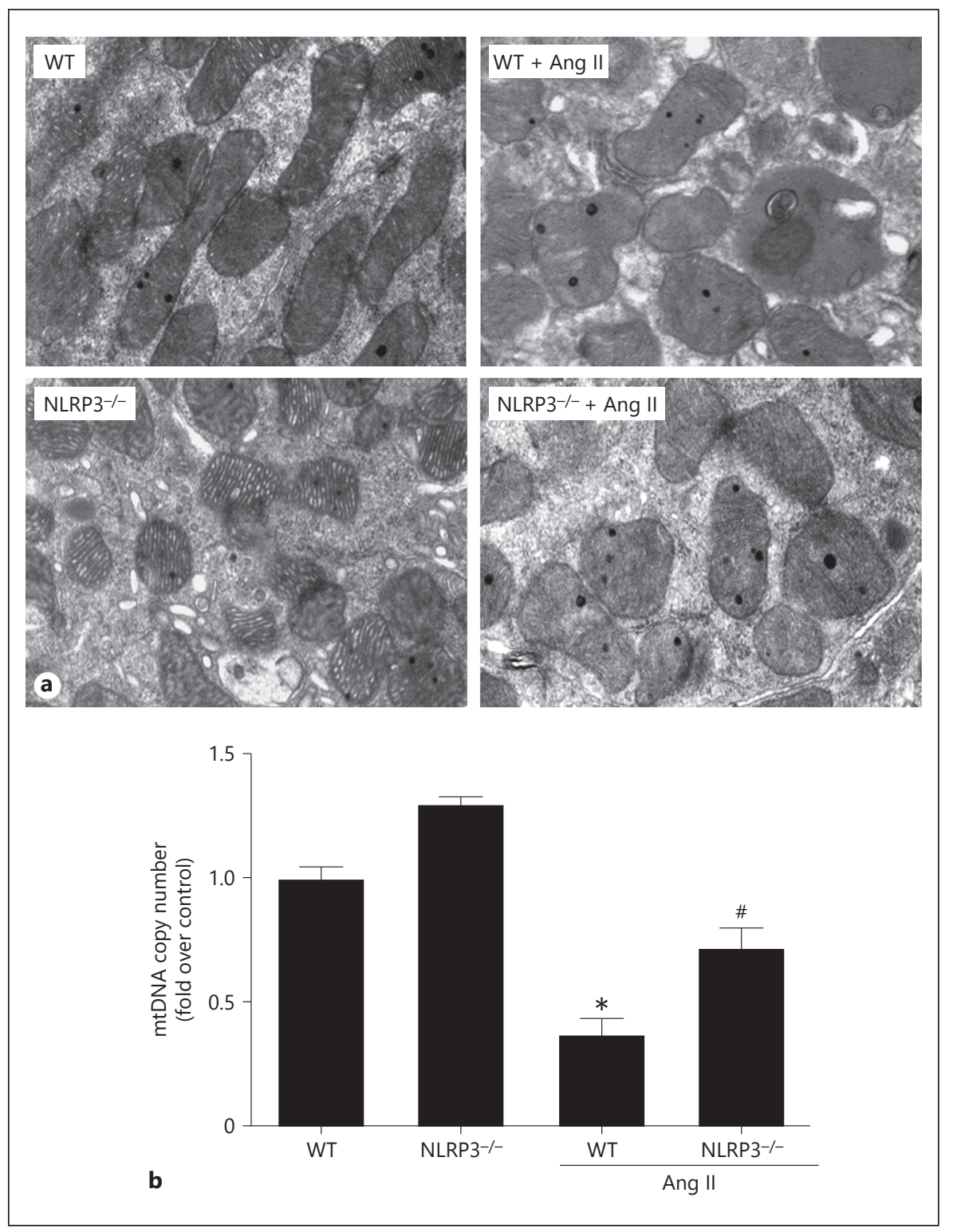

down of NLRP3 by a siRNA approach in podocytes remarkably mitigated the mitochondrial abnormalities. In animals, as expected, systemic deletion of NLRP3 also significantly attenuated mitochondrial dysfunction. These results suggest that Ang II-induced NLRP3 inflammasome activation could promote mitochondrial dysfunction in podocytes.

In addition, the blood pressure was similarly increased in WT and NLRP3 ${ }^{-/-}$mice after Ang II infusion, with no difference between genotypes, which is in agreement with the data reported by Wen et al. [28]. This result suggests that the attenuation of podocyte injury and mitochon- drial dysfunction in NLRP3 ${ }^{-/-}$mice is independent of the hypertension effect.

In summary, in the present study, we presented new evidence showing that activation of the NLRP3 inflammasome mediated Ang II-induced podocyte injury, possibly through inducing mitochondrial dysfunction. Based on the importance of NLRP3 inflammasome activation in the development and progression of renal diseases, our findings emphasize the importance of targeting the NLRP3 inflammasome and/or mitochondria to develop novel strategies for the prevention and treatment of podocytopathy. 


\section{Acknowledgments}

This work was supported by grants from the National Key Research and Development Program (No. 2016YFC0906103), the National Natural Science Foundation of China (No. 81530023, 81570616, and 81470928), and the Natural Science Foundation of Jiangsu Province (No. BK20141079 and BL2014007).

\section{Statement of Ethics}

All animal experiments were performed in accordance with the guidelines and regulations of the Animal Experimentation Ethics Committee of Nanjing Medical University. All experimental protocols were approved by the same committee.

\section{Disclosure Statement}

The authors have declared that no competing interest exists.

\section{Author Contributions}

M. Zhao and M. Bai performed the experiments and wrote the manuscript; G. Ding, Y. Zhang, S. Huang, and Z. Jia helped design the experiments and interpreted the data; A. Zhang designed the experiments, interpreted the data, wrote the manuscript, and approved the final version of the manuscript for publication.

\section{References}

1 Li XC, Zhang J, Zhuo JL: The vasoprotective axes of the renin-angiotensin system: physiological relevance and therapeutic implications in cardiovascular, hypertensive and kidney diseases. Pharmacol Res 2017;125(pt A): 21-38.

2 Horita S, Nakamura M, Shirai A, Yamazaki O, Satoh N, Suzuki M, Seki G: Regulatory roles of nitric oxide and angiotensin II on renal tubular transport. World J Nephrol 2014;3:295301.

-3 Yang Y, Yang Q, Yang J, Ma Y, Ding G: Angiotensin II induces cholesterol accumulation and injury in podocytes. Sci Rep 2017;7: 10672.

-4 Rizzo P, Novelli R, Rota C, Gagliardini E, Ruggiero B, Rottoli D, Benigni A, Remuzzi G: The role of angiotensin II in parietal epithelial cell proliferation and crescent formation in glomerular diseases. Am J Pathol 2017;187: 2441-2450.

5 Xu Z, Li W, Han J, Zou C, Huang W, Yu W, Shan X, Lum H, Li X, Liang G: Angiotensin II induces kidney inflammatory injury and fibrosis through binding to myeloid differentiation protein-2 (MD2). Sci Rep 2017;7: 44911.

6 Sharma R, Kinsey GR: Regulatory T cells in acute and chronic kidney diseases. Am J Physiol Renal Physiol 2017, Epub ahead of print.

-7 Lee SB, Kalluri R: Mechanistic connection between inflammation and fibrosis. Kidney Int Suppl 2010;119:S22-S26.

8 Meng XM, Nikolic-Paterson DJ, Lan HY: Inflammatory processes in renal fibrosis. Nat Rev Nephrol 2014;10:493-503.

-9 Lv LL, Liu BC: Role of non-classical reninangiotensin system axis in renal fibrosis. Front Physiol 2015;6:117.

10 Tschopp J, Schroder K: NLRP3 inflammasome activation: the convergence of multiple signalling pathways on ROS production? Nat Rev Immunol 2010;10:210-215.
11 Patel S: Inflammasomes, the cardinal pathology mediators are activated by pathogens, allergens and mutagens: a critical review with focus on NLRP3. Biomed Pharmacother 2017;92:819-825.

12 Zhuang Y, Ding G, Zhao M, Bai M, Yang L, Ni J, Wang R, Jia Z, Huang S, Zhang A: NLRP3 inflammasome mediates albumin-induced renal tubular injury through impaired mitochondrial function. J Biol Chem 2014; 289:25101-25111.

13 Bai M, Chen Y, Zhao M, Zhang Y, He JC, Huang S, Jia Z, Zhang A: NLRP3 inflammasome activation contributes to aldosteroneinduced podocyte injury. Am J Physiol Renal Physiol 2017;312:F556-F564.

14 Liu D, Xu M, Ding LH, Lv LL, Liu H, Ma KL, Zhang AH, Crowley SD, Liu BC: Activation of the Nlrp3 inflammasome by mitochondrial reactive oxygen species: a novel mechanism of albumin-induced tubulointerstitial inflammation. Int J Biochem Cell Biol 2014;57:7-19.

15 Fu R, Guo C, Wang S, Huang Y, Jin O, Hu H, Chen J, Xu B, Zhou M, Zhao J, Sung SJ, Wang H, Gaskin F, Yang N, Fu SM: Podocyte activation of NLRP3 inflammasomes contributes to the development of proteinuria in lupus nephritis. Arthritis Rheumatol 2017;69:16361646.

16 Che R, Yuan Y, Huang S, Zhang A: Mitochondrial dysfunction in the pathophysiology of renal diseases. Am J Physiol Renal Physiol 2014;306:F367-F378.

17 Yuan Y, Chen Y, Zhang P, Huang S, Zhu C, Ding G, Liu B, Yang T, Zhang A: Mitochondrial dysfunction accounts for aldosteroneinduced epithelial-to-mesenchymal transition of renal proximal tubular epithelial cells. Free Radic Biol Med 2012;53:30-43.
18 Kadoguchi T, Kinugawa S, Takada S, Fukushima A, Furihata T, Homma T, Masaki Y, Mizushima W, Nishikawa M, Takahashi M, Yokota T, Matsushima S, Okita K, Tsutsui H: Angiotensin II can directly induce mitochondrial dysfunction, decrease oxidative fibre number and induce atrophy in mouse hindlimb skeletal muscle. Exp Physiol 2015; 100:312-322.

19 Mundel P, Reiser J, Kriz W: Induction of differentiation in cultured rat and human podocytes. J Am Soc Nephrol 1997;8:697-705.

20 Acton BM, Jurisicova A, Jurisica I, Casper RF: Alterations in mitochondrial membrane potential during preimplantation stages of mouse and human embryo development. Mol Hum Reprod 2004;10:23-32.

21 Greka A, Mundel P: Cell biology and pathology of podocytes. Annu Rev Physiol 2012;74: 299-323.

22 Urushihara M, Kagami S: Role of the intrarenal renin-angiotensin system in the progression of renal disease. Pediatr Nephrol 2017;32:1471-1479.

23 Della Penna SL, Rosón MI, Toblli JE, Fernández BE: Role of angiotensin II and oxidative stress in renal inflammation by hypernatremia: benefits of atrial natriuretic peptide, losartan, and tempol. Free Radic Res 2015;49: 383-396.

24 Chang A, Ko K, Clark MR: The emerging role of the inflammasome in kidney diseases. Curr Opin Nephrol Hypertens 2014;23:204-210.

25 Lorenz G, Darisipudi MN, Anders HJ: Canonical and non-canonical effects of the NLRP3 inflammasome in kidney inflammation and fibrosis. Nephrol Dial Transplant 2014; $29: 41-48$

26 Vilaysane A, Chun J, Seamone ME, Wang W, Chin R, Hirota S, Li Y, Clark SA, Tschopp J, Trpkov K, Hemmelgarn BR, Beck PL, Muruve DA: The NLRP3 inflammasome promotes renal inflammation and contributes to CKD. J Am Soc Nephrol 2010;21:1732-1744. 
27 Gong W, Mao S, Yu J, Song J, Jia Z, Huang S, Zhang A: NLRP3 deletion protects against renal fibrosis and attenuates mitochondrial abnormality in mouse with 5/6 nephrectomy. Am J Physiol Renal Physiol 2016;310:F1081F1088.

28 Wen Y, Liu Y, Tang T, Lv L, Liu H, Ma K, Liu B: NLRP3 inflammasome activation is involved in Ang II-induced kidney damage via mitochondrial dysfunction. Oncotarget 2016; 7:54290-54302.
9 Zhang GX, Lu XM, Kimura S, Nishiyama A: Role of mitochondria in angiotensin II-induced reactive oxygen species and mitogenactivated protein kinase activation. Cardiovasc Res 2007;76:204-212.

30 Herrera M, Silva GB, Garvin JL: Angiotensin II stimulates thick ascending limb superoxide production via protein kinase $\mathrm{Ca}$-dependent $\mathrm{NADPH}$ oxidase activation. J Biol Chem 2010;285:21323-21328.
31 Tsai IC, Pan ZC, Cheng HP, Liu CH, Lin BT, Jiang MJ: Reactive oxygen species derived from NADPH oxidase 1 and mitochondria mediate angiotensin II-induced smooth muscle cell senescence. J Mol Cell Cardiol 2016; 98:18-27.

32 Zhuang Y, Yasinta M, Hu C, Zhao M, Ding G, Bai M, Yang L, Ni J, Wang R, Jia Z, Huang S, Zhang A: Mitochondrial dysfunction confers albumin-induced NLRP3 inflammasome activation and renal tubular injury. Am J Physiol Renal Physiol 2015;308:F857-F866. 